\title{
Für die Flora Bulgariens neue und seltene Pflanzen.
}

Von N. Stojanov und B. Stefanov (Sofia).

Aira capillaris Host var. ambigua Not. Strandja, an Felsen bei dem Dorfe Kosti.

Poa caesia Sm. An Felsen bei Sara-Gjol (Rila) und Resnovete (Vitoša). Festuca Lachenalii Spenn. var. mutica Asch. et Graebn. Strandja, an Felsen bei Kosti.

Scirpus triqueter L. Auf Sand am Meeresstrande bei Mesemvria.

Schoenus nigricans L. Auf Sand am Meeresstrande bei Burgas.

Carex prolixa Fries. An dem Sumpfe bei Dragoman.

Allium nigrum L. Auf Ackerfeldern bei Nova Machla (Bezirk Philippopel), gesammelt von Střibrny. Bis jetzt nur am Ali-Botuš in OstMazedonien gefunden.

- Cyrilli Ten. Am Rande der Ackerfelder bei dem Dorfe Poturnak (Strandja).

Nectaroscordum siculum Ucria var. Dioscoridis Richt. In schattigen Wäldern bei Küprija (Strandja). Innerhalb der Grenzen Bulgariens nur für die Umgebung von Varna bekannt (Davidov).

Tulipa australis Lev. An den Hügeln Bakardjik bei Jambol. Diese Art scheint in Bulgarien ziemlich weit verbreitet zu sein, wird aber öfters mit $T$. silvestris verwechselt, zu der sie auch Übergangsformen bildet.

Scilla bithynica Boiss. (S. Radkae Davidor, in Magyar Botanikai Lapok, 1905.) In schattigen Uferwäldern an dem Flusse Kargana, südlich von Sozopol.

Ornithogalum Wiedemanni Boiss. An dem Strandja-Gebirge, in Gebüschen bei Malko-Tirnovo und Siva.

Gladiolus paluster Gaud. Áli-Botuš, an grasigen Stellen auf ČengeneKale $(1300 \mathrm{~m})$.

Orchis provincialis Balb. Strandja, in Gebüschen bei Kosti und nördlich von Pergopla.

Salix retusa L. Auf Felsen des Gipfels Ibar $(2500 \mathrm{~m})$ an dem RilaGebirge und auf dem Sinanica-Gipfel an dem Pirin.

Moenchia quaternella Ehrh. An feuchtem Lehmboden bei Poda, südlich von Burgas; selten.

Arenaria graeca Boiss. Auf Felsen in der Schlucht Jundol (Rhodopen). Anemone pavonina Lam. (A. fulgens Gay) typica. In Gebuschen und lichten Wäldern bei Küprija und Siva (Strandja). 
Anemone pavonina Lam. var. purpureo-violacea Boiss. Südlich von Burgas und überall an dem Strandja-Gebirgè. Hieher gehören auch die Pflanzen von Chaskovo in dem Herbarium Georgie vs.

Ranunculus chaerophyllus L. (R. peloponnesiacus Boiss.). In Gebüschen und auf Wiesen sũdlich von Burgas und bei Malko-Tirnovo.

Epimedium pubigerum Mor. et Dec. In schattigen Wäldern bei Urgari und nördlich von Pergopla, an dem Strandja-Gebirge.

Cardamine pratensis L. var. dentata Koch. Auf feuchten und schattigen Stellen bei Kuprija, Urgari und Kosti, an dem Strandja-Gebirge.

Prunus laurocerasus L. In tiefen, von Fagus orientalis bewaldeten Schluchten bei Kosti an dem Strandja-Gebirge in Gesellschaft von Rhododendron ponticum, Daphne pontica, Ilex aquifolium und Ruscus hypoglossum. Bis jetzt war diese Pflanze in Bulgarien nur von dem Sipka- und Gabrovo-Balkan bekannt.

Hymenocarpus circinatus (L.) Sav. In Gebüschen südlich von Sozopol. Astragalus testiculatus Pall. Auf Kalkfelsen am Čepan bei Dragoman.

- pubiflorus DC. Auf Kalkfelsen bei dem Dorfe Malo-Malovo (Bezirk Sofia). Bis jetzt nur für Ostbulgarien angegeben (Davidov).

- australis (L.) Lam. (Phaca australis L.). Auf Felsen bei Edi-Gjol (2500 m) an dem Rila-Gebirge.

Vicia hybrida L. In Gebüschen und Weingärten bei Sozopol.

- Barbazitae Ten. In Gebüschen und lichten Wäldern; verbreitet an dem Strandja-Gebirge und bei Sozopol. Bis jetzt nur an dem BelasicaGebirge gefunden.

Ervum lenticula (Schreb.) Alef. An Kalkfelsen des Strandja-Gebirges bei Malko-Tirnovo.

Polygala supina Schreb. Verbreitet in Gebüschen und lichten Wäldern an dem Strandja-Gebirge. Für den Vratca-Balkan in Westbulgarien angegeben (Velenorsky).

Hypericum androsaemum L. In schattigen Wäldern von Fagus orientalis bei Kosti an dem Strandja-Gebirge in der Gesellschaft von Rhododendron ponticum, Daphne pontica, Ilex aquifolium u. dgl.

Euphorbia apios L. var. lamprocarpa Boiss. In Gebüschen bei Č́engeneSkele, südlich von Burgas.

- thyrsiflora Griseb. (Spicil. I, p. 143-144). In Gebüschen zwischen Burgas und Sozopol. Diese Pflanze haben wir schon vorher am Tekir-Dagh (loco classico) gesammelt und halten sie für eine Form, die mit $E$. agraria MB. nicht identisch ist, aber vielleicht am besten als eine Varietät der letzteren Art betrachtet werden kann. Im Vergleich mit der in Bulgarien verbreiteten $E$. agraria MB. ist diese Form ansehnlich größer, mit großen, abgerundeten Blättern und unterOsterr. botan. Zeitschrift, 1921, Heft 9-12. 
scheidet sich weiter auch durch die anderen, von Grisebach (1. c., p. 144) richtig angegebenen Merkmale.

Tamarix tetrandra Pall. Am Meeresstrande südlich von Burgas.

Erica arborea L. An trockenen Hügeln bei Kosti (Strandja) in der Gesellschaft von Calluna vulgaris, Cistus creticus, Hypericum rhodopeum u. a.

Cyclamen coum Mill. Verbreitet in den Wäldern an dem StrandjaGebirge. Diese Art scheint nur in Ostbulgarien verbreitet zu sein. Bis jetzt wurde sie aus der Umgebung von Sumen, Tirnovo und Sliven bekannt.

Symphytum tauricum Willd. An feuchtem Gesteine bei Kosti (Strandja). Sideritis taurica MB. An trockenen Kalkfelsen des Strandja-Gebirges bei Malko-Tirnovo.

Salvia officinalis L. An steinigem Boden bei Poda, südlich von Burgas (vielleicht verwildert).

Pedicularis scardica G. Beck. Auf Kalkfelsen bei Dragoman und Belidie; auch an dem Koneva-Gebirge (Bezirk Küstendil).

\section{Campanula Cenisia L. in den Zillertaler Alpen.}

Von Dr. Heinrich Handel-Mazzetti (Wien).

Im vergangenen Sommer erzählte mir mein Bruder Hermann, daß sein Freund Dr. Hermann Lechner in Hall in Tirol ihm verblühte Exemplare von Campanula Cenisia aus dem Wattentale vorgelegt habe. Da ein Vorkommen dieser Pflanze dort außerordentlich merkwürdig und eine Verwechslung mit Exemplaren aus den vom Sammler kurz vorher besuchten Rhätikon in West-Tirol nicht ausgeschlossen schien, suchte mein Bruder am 21. Oktober 1921 den angegebenen Standort auf und fand Campanula Cenisia tatsächlich auf dem östlichsten der Tarntaler Köpfe wieder. Diese Berggruppe gehört zu der Tuxer Gruppe der Zillertaler Alpen und liegt am Knotenpunkte des bei Matrei in die Sill mündenden Navistales, des Wattentales, welches ins Unterinntal mündet, und des Tuxertales, des westlichsten oberen Seitentales des Zillertales. Ihre geologische Mannigfaltigkeit bedingt einen großen Reichtum der. Flora, der schon von Kerner aufgeschlossen wurde. Der östlichste Gipfel, Pluderling genannt, war aber offenbar noch von keinem Botaniker besucht worden. Mein Bruder schreibt mir über das Vorkommen folgendes: „Der Standort befindet sich östlich des Ostgipfels des Pluderling (Kote $2756 \mathrm{~m})$. Der Berg fällt nach Süden in steilen Brennerphyllitfelsen ab. 